\title{
First Data from the HERMES Recoil Detector
}

Nils Pickert for the HERMES-Collaboration

<pickert@pi2.physik.uni-erlangen.de>

Physikalisches Institut 2, Universität Erlangen-Nürnberg, Erwin-Rommel-Straße 1, 91058 Erlangen, Germany

\section{Physics Motivation}

The HERMES-Recoil detector detects particles unde large angles originating from exclusive reactions, e. Deeply Virtual Compton Scattering (DVCS), the hard exclusive lepto-production of a real photon.

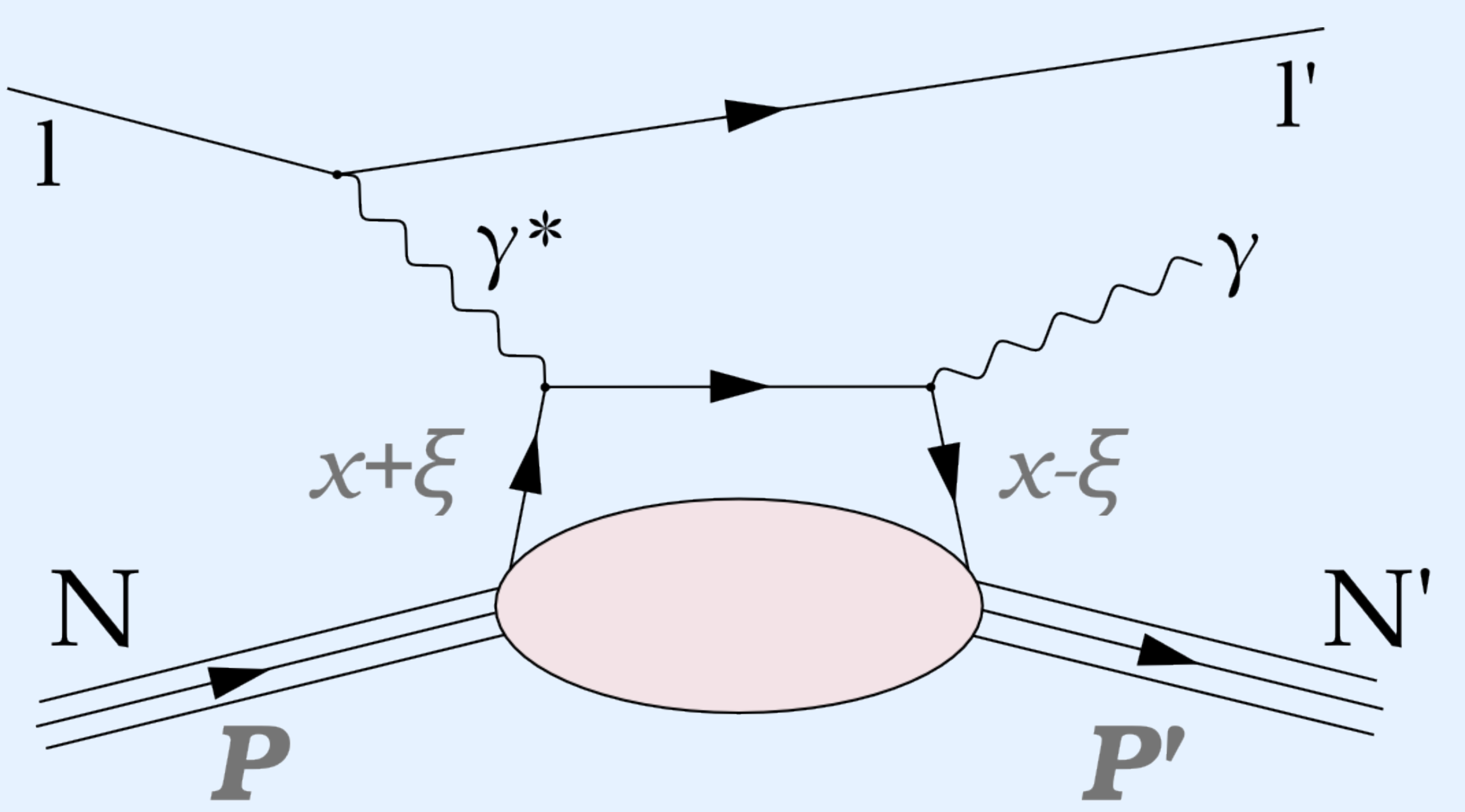

In the energy range accessible by HERMES the cross section is dominated by Bethe-Heitler (or Bremsstrahlung-) processes, but the interference with DVCS leads to measurable beam charge and beam spin asymmetries, from which information on Generalised Parton Distributions (GPDs) can be obtained.

\section{SD: Correlated Noise}

The electromagnetic fields generated by the beam induce a high amount of correlated noise and common mode noise (CMN). A reduction is already done on-line by the ADCs using the average value of the first 16 strips, but as the correlation is not linear over a sensor, an additional spline fitting method is used. A MIP can be detected with signal to noise ratio of around 5:1 in the HG chain. The maximum deposited energy by a proton is $6.2 \mathrm{MeV}$ (eq. $60 \mathrm{MIPs}$ ) with a SNR of $250: 1$ in the LG chain.

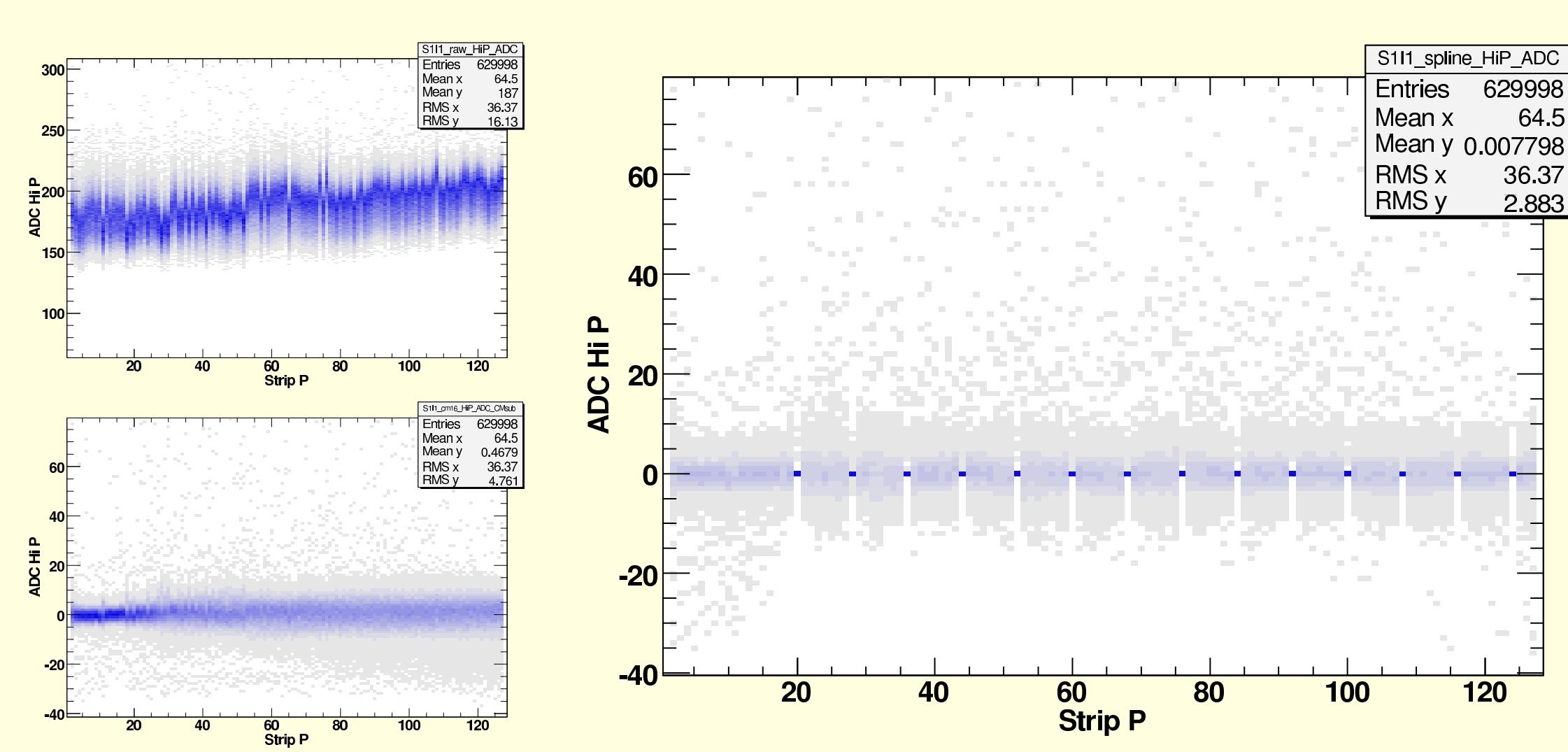

top left: raw data spectrum

bottom left: data after online common mode subtraction right: data after spline common mode subtraction

\section{SD: Energy Loss}

Proton momenta can be determined over a large kinematical range from the energy deposition in the sensors.

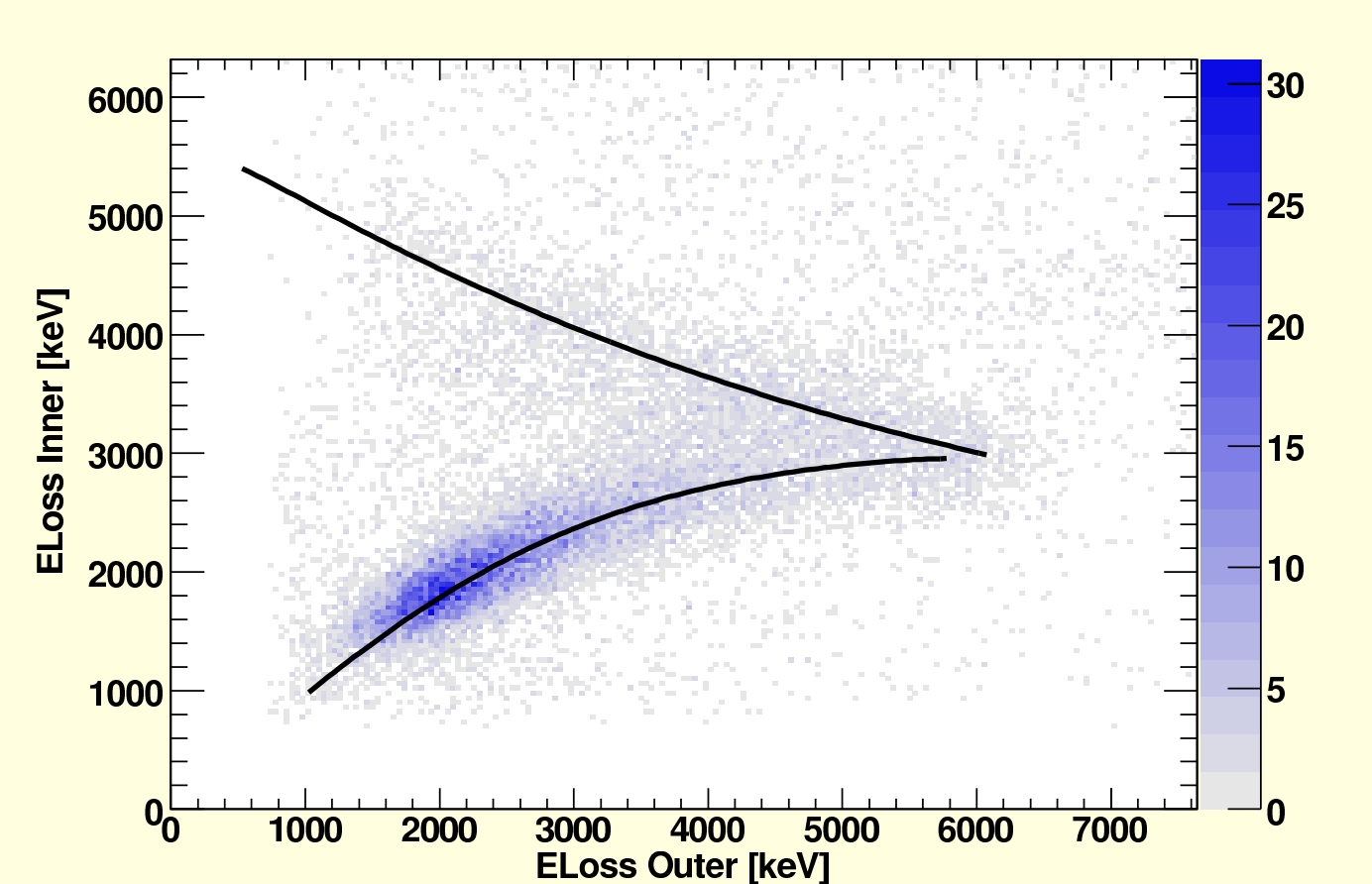

Histogramm: energy loss in the inner silicon layer versus energy loss in the outer layer.

Continuous lines: calculated energy loss (SRIM)

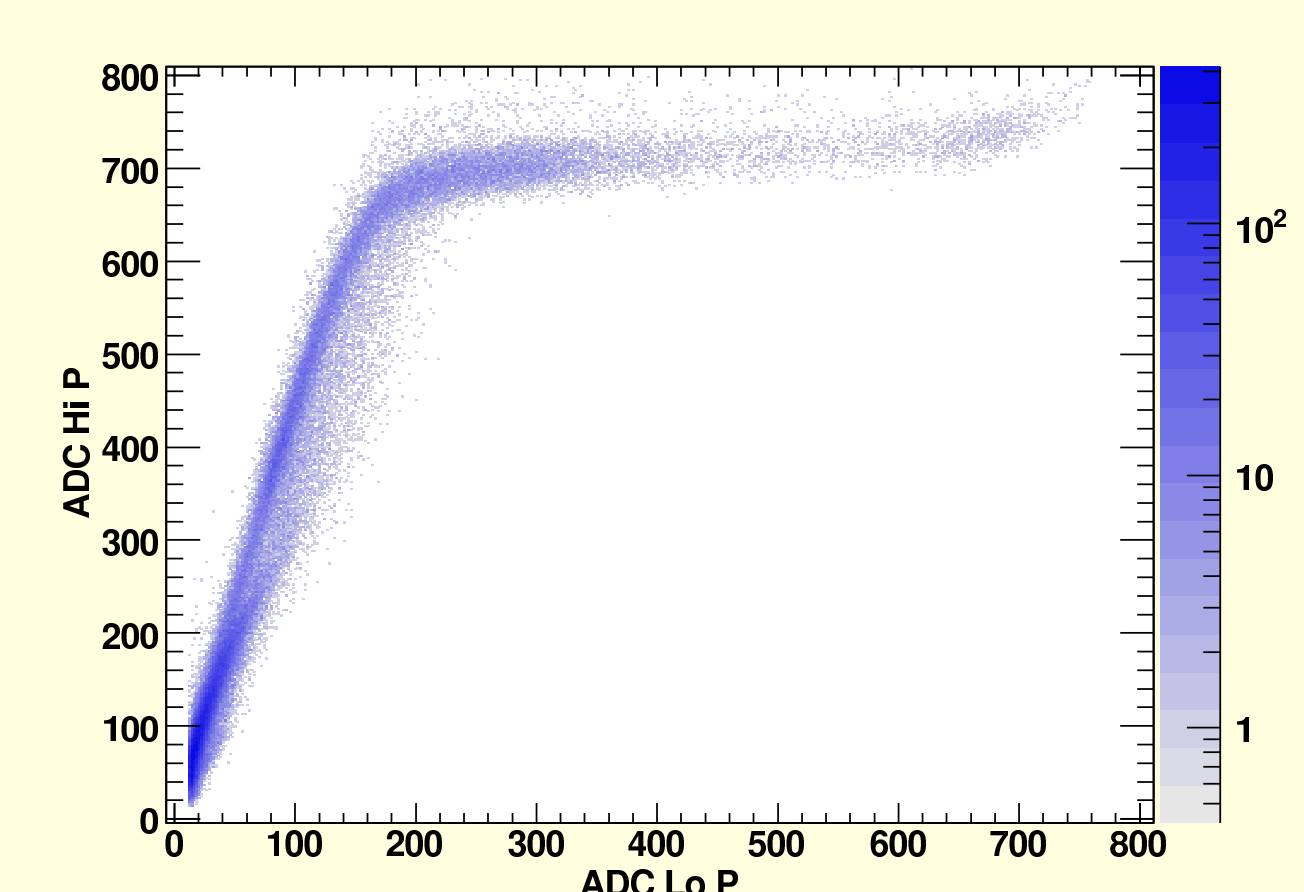

High gain signal versus low gain signal. At around 700 ADC channels the high gain Helix is saturated.

\section{The Recoil Detector}

A Silicon Detector (SD), located inside the HERA vacuum. Consisting of 16 double sided TIGRE sensors measuring $10 \mathrm{~cm} \times 10 \mathrm{~cm}, 128$ strips per sensor-side.

A Scintillating Fibre Detector (SF) consisting of 2 barrels made of two layers of $1 \mathrm{~mm}$ Kuraray fibres, 5120 channels in total.

A Photon Detector (PD) consisting of three layers of scintillator bars with tungsten as converter.

A superconducting magnet, providing a longitudinal field of $1 \mathrm{~T}$ strength.

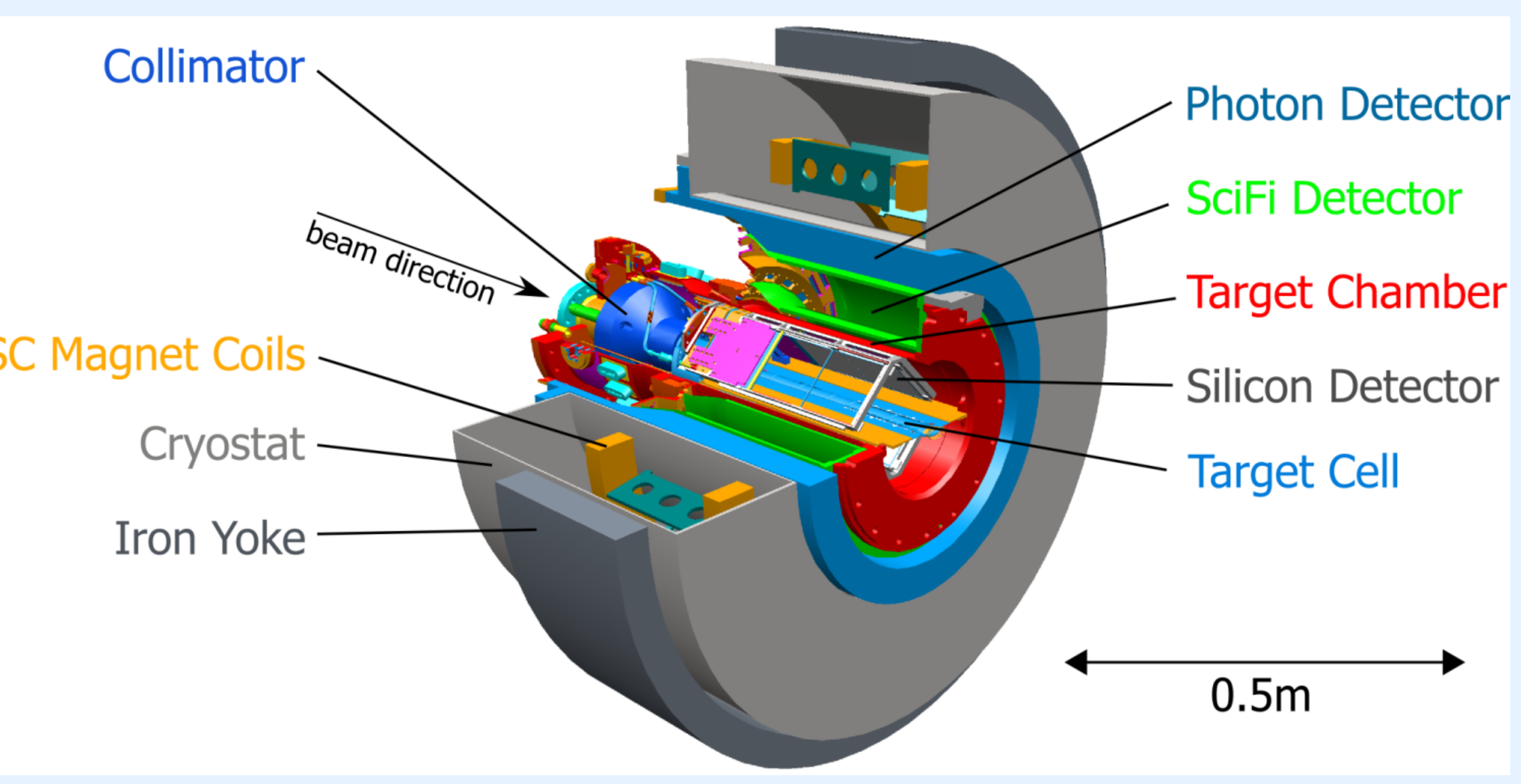

The Recoil detector was installed in December 2005 It is taking data since the beginning of 2006 and will continue until June 2007.

\section{SD: Silicon only tracking}

Tracks can be reconstructed from the silicon information only.

Reconstructed z-Vertex:

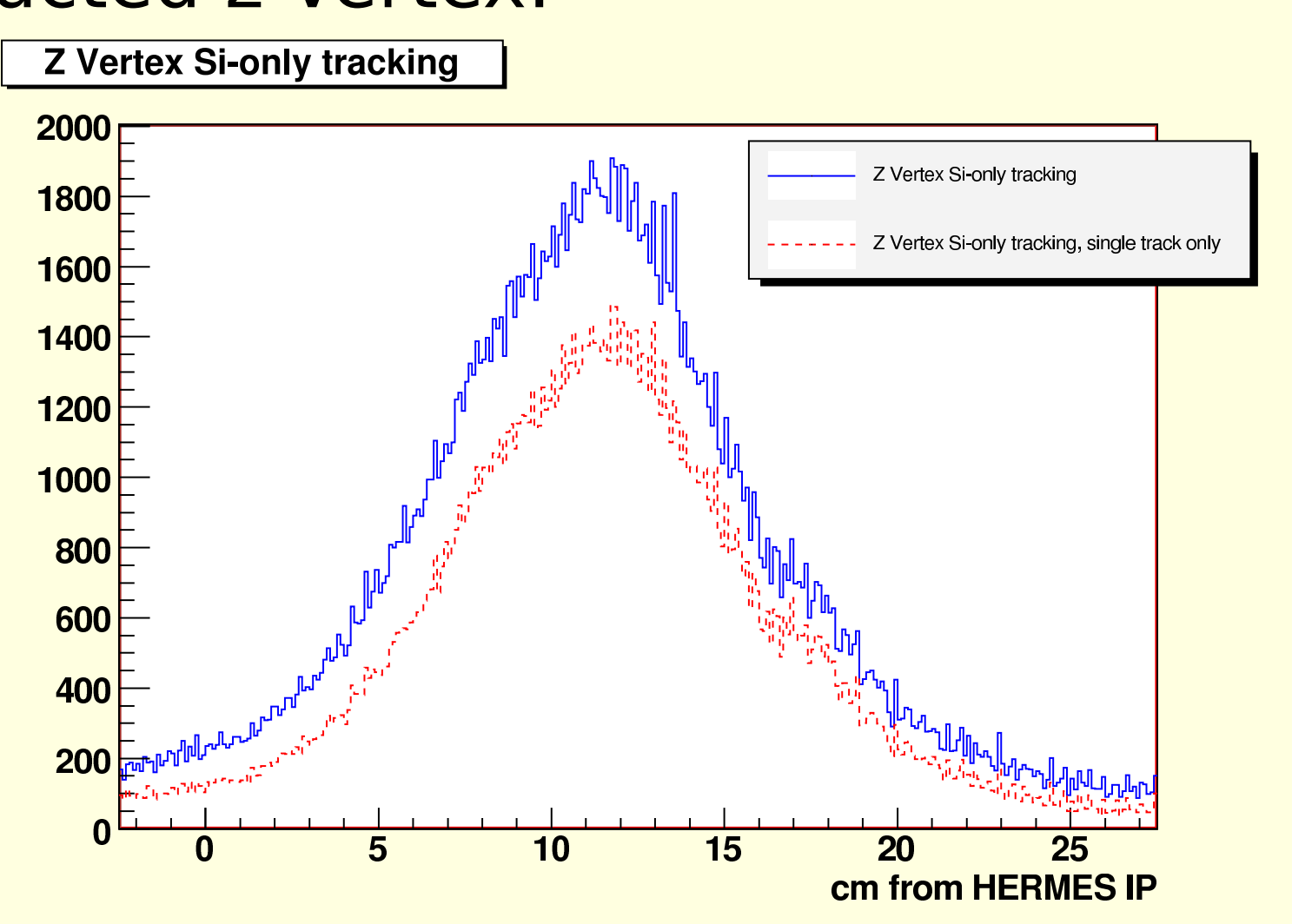

The polar angle $\theta_{P}$ of the scattered proton determined with silicon only tracking versus the momentum of the scattered lepton determined by the spectrometer: Protons are detected in a polar angular range $0.2 \mathrm{rad}<\theta_{p}<2.8 \mathrm{rad}$

Data:

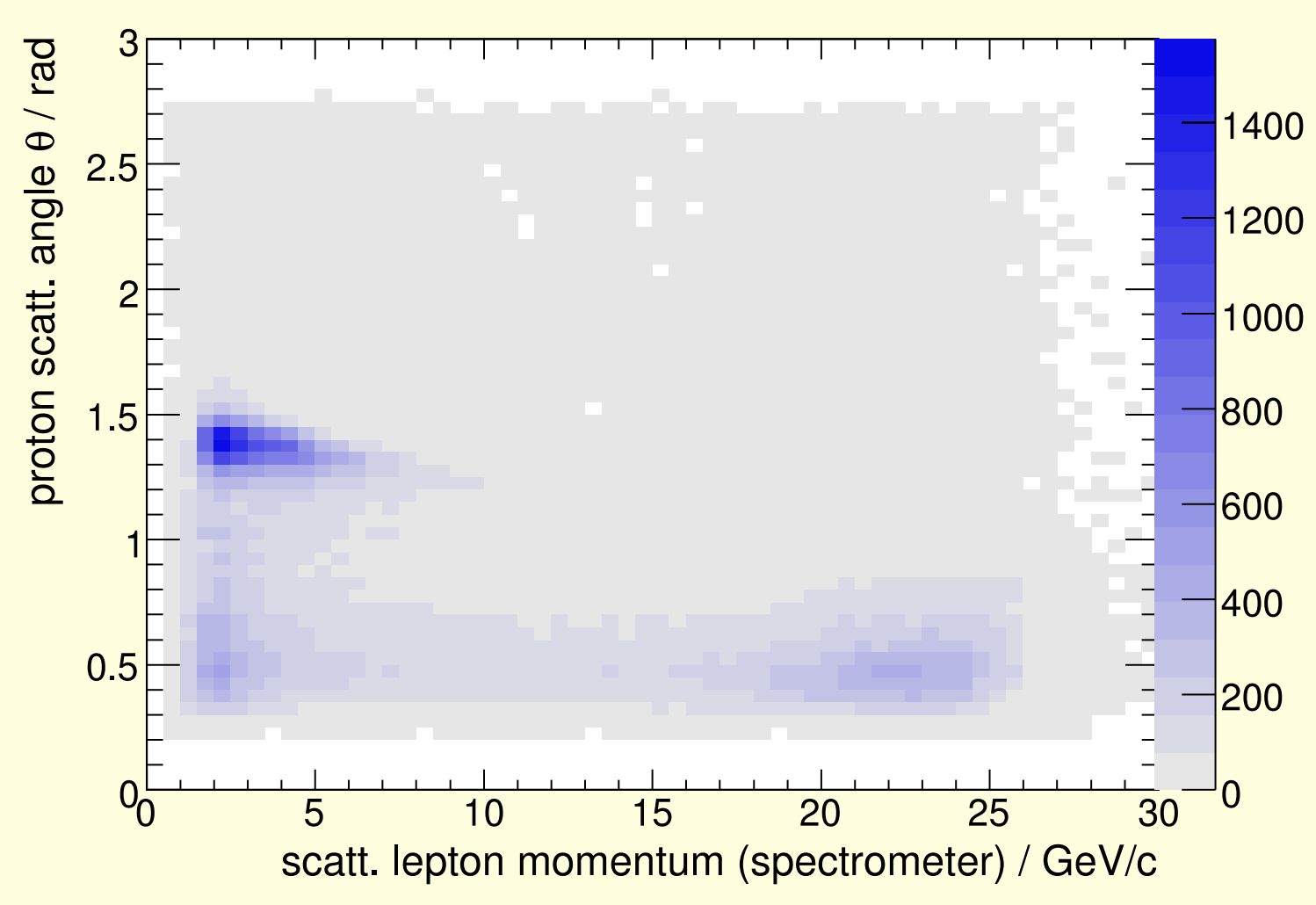

Monte Carlo Simulation:
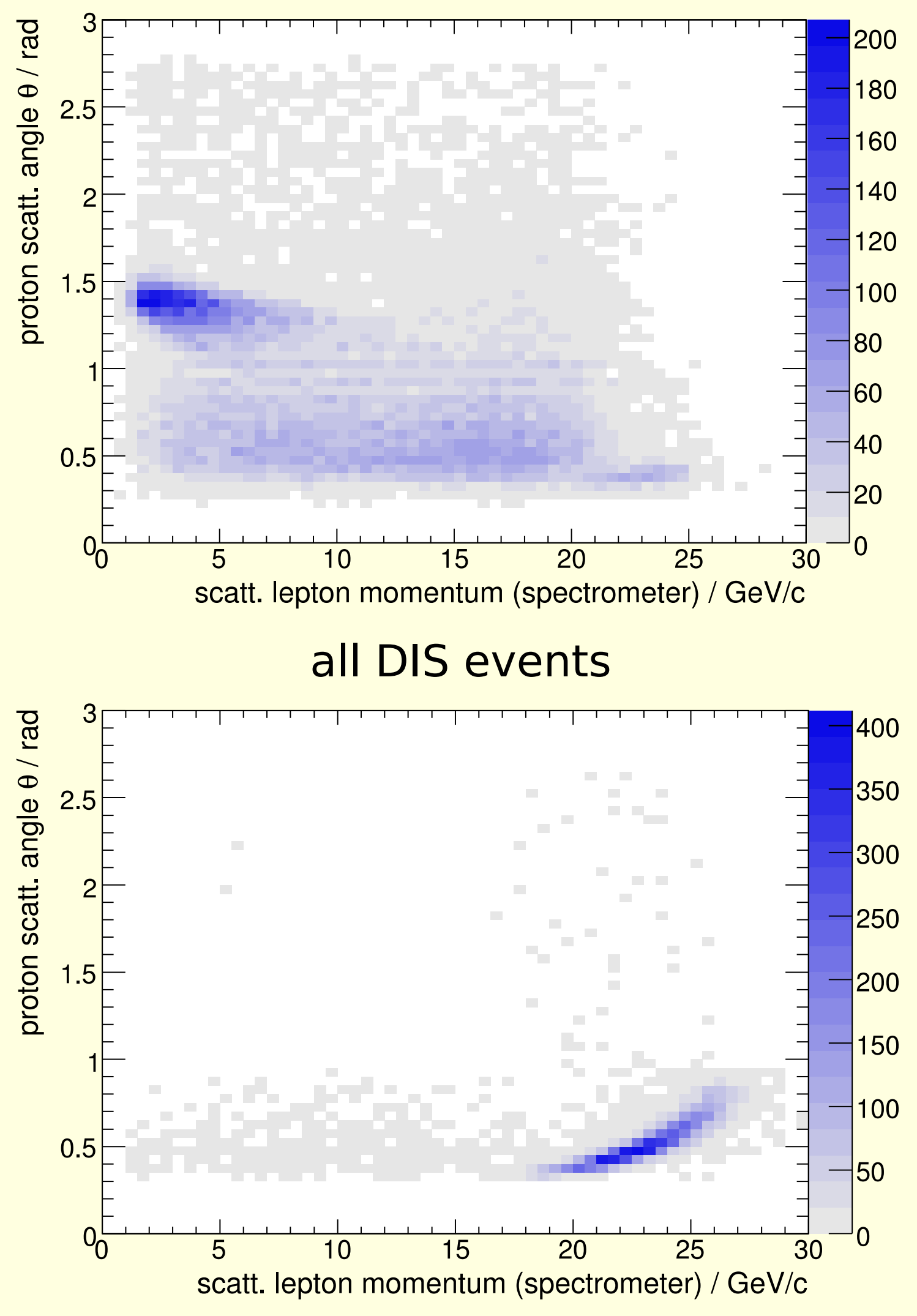

elastic e-p-scattering

Elastic e-p events can be kinematically separated from DIS events.

\section{The Silicon Subdetector}

The silicon detector features 16 sensors, arranged in two layers of modules containing two sensors. These modules are arranged in a square tube around the target cell.

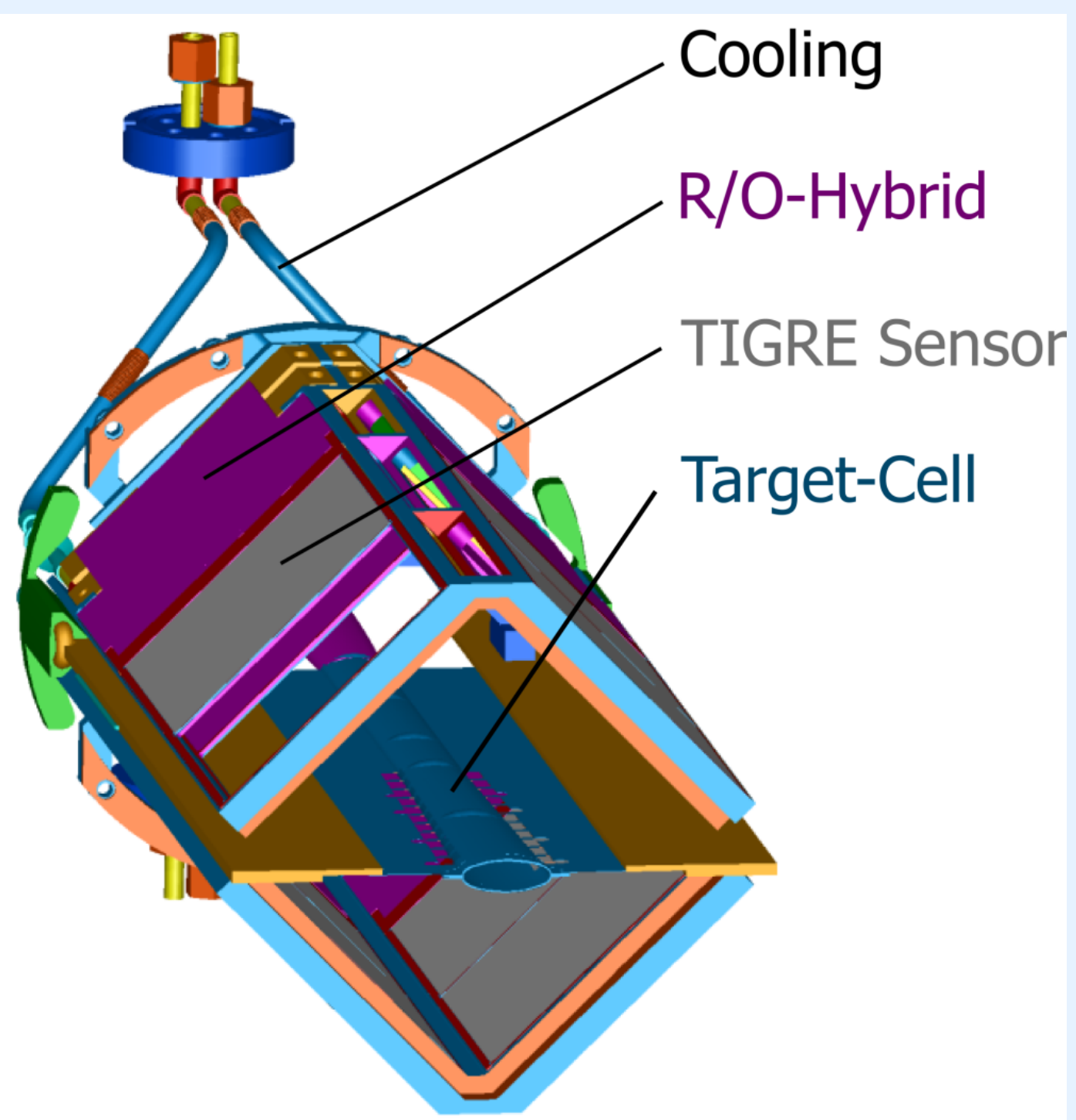

To provide the necessary dynamic range from $<1$ to 70 MIPs (Minimum lonising Particle), a charge-sharing readout has been devised. Each detector strip is read out by two chips, one connected directly, the other via a $10 \mathrm{pF}$ capacitor.

\section{SF: SciFi only tracking}

Elastic e-p scattering

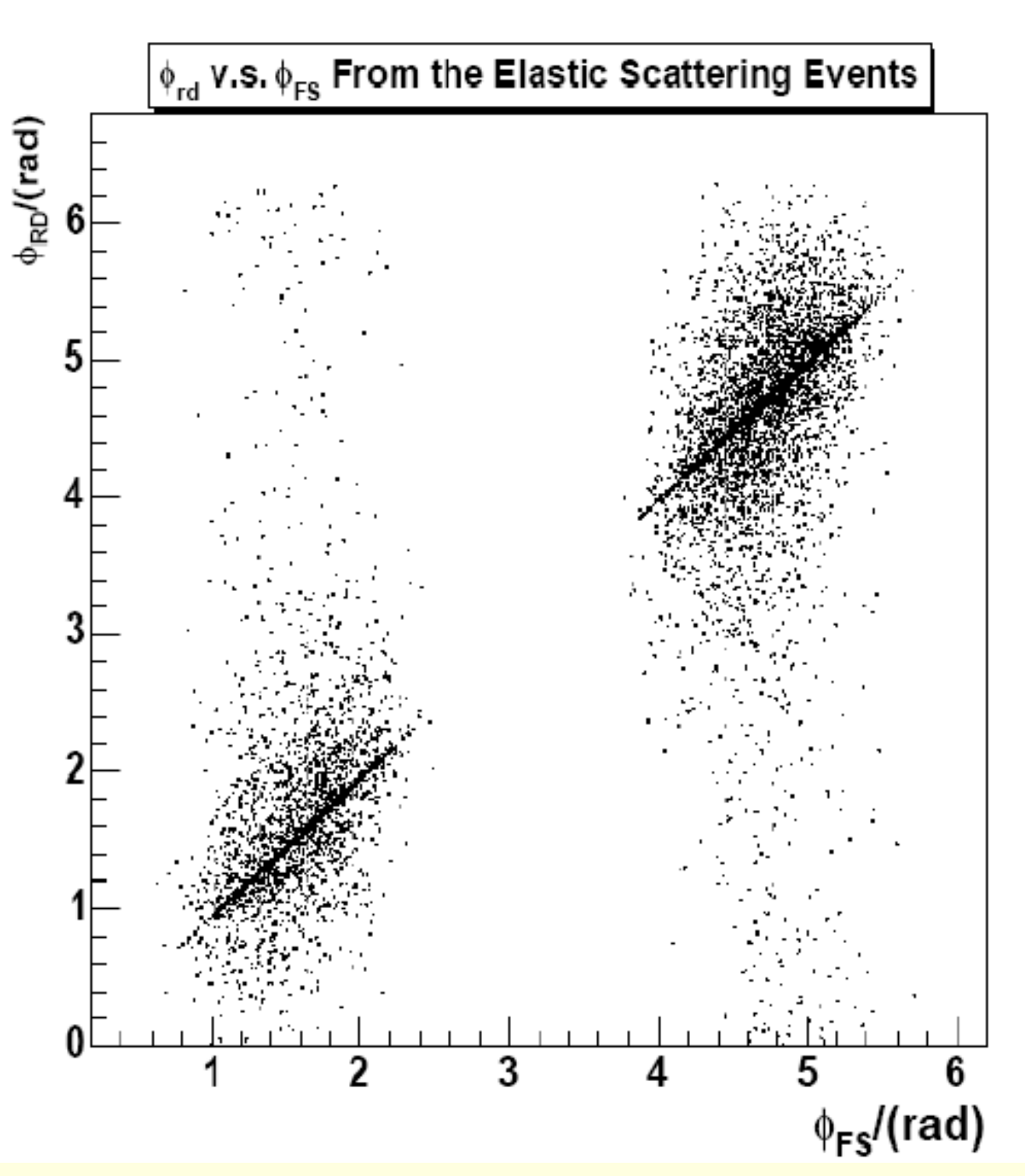

Azimuthal proton scattering angle $\phi$ measured by the SciFi versus the same angle calculated from the lepton detected in the forward spectrometer. The angular resolution of the SciFi is $8 \mathrm{mrad}$. The gap results from the spectrometer septum plate.

\section{SF: Background suppression}

The Scintillating Fibre tracker is read out with electronics based on the GASSIPLEX chip. The chip's integration time covers multiple HERA bunch crossings, so each read out data set also contains hits from bunches before and after the triggered event.

Using a TDC to obtain fast timing information from the photo multipliers it is possible to select the hits from a single bunch crossing. The average multiplicity for each layer is around 4.

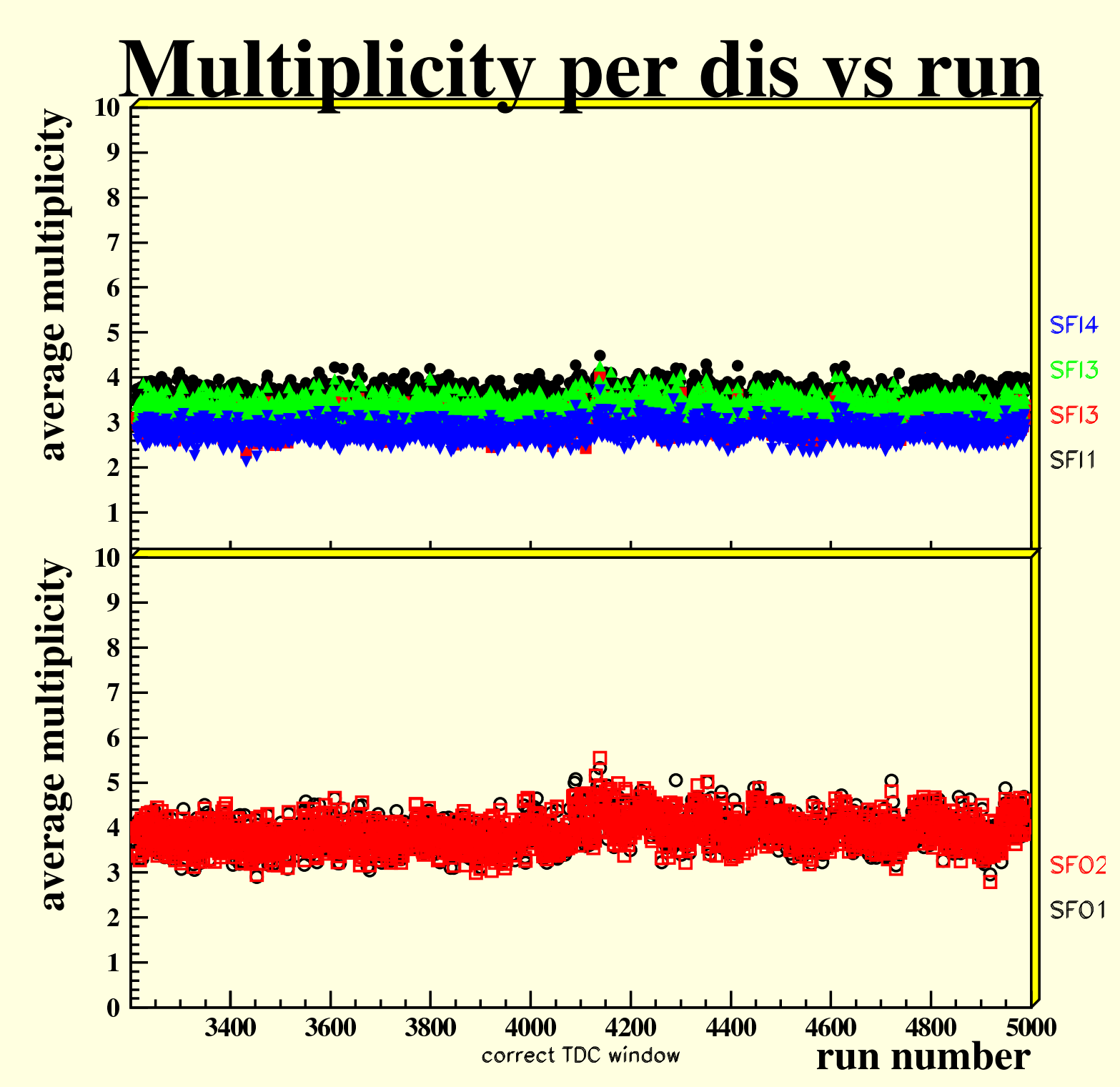

